\title{
Large homogeneous sample of X-ray selected AGN and its study
}

\author{
Areg Mickaelian, Gurgen Paronyan and Hayk Abrahamyan \\ Byurakan Astrophysical Observatory (BAO), Byurakan 0213, Aragatzotn Province, Armenia \\ email: aregmick@yahoo.com
}

\begin{abstract}
The combined catalogue of AGN selected from optical identifications of X-ray sources based on Hamburg-ROSAT and Byurakan-Hamburg-ROSAT catalogues is a homogeneous sample for statistical studies. Optically identified X-ray sources from ROSAT BSC and FSC are included, $4253 \mathrm{X}$-ray selected AGN in total. We carried out classification for 210 of these candidate sources based on available SDSS spectra and enlarged the sample of confirmed AGN. Statistical investigations of the sample were also carried out. Multiwavelength SEDs have also been constructed to link them to classifications.
\end{abstract}

Keywords. galaxies: nuclei, galaxies: Seyfert, galaxies: statistics, X-rays: galaxies

Based on Hamburg-ROSAT Catalogue (HRC; Zickgraf et al. 2003) and ByurakanHamburg-ROSAT Catalogue (BHRC; Mickaelian et al. 2006), a combined homogeneous catalogue of AGN (ROSAT BSC/FSC AGN) selected from optical identifications of Xray sources was created for statistical studies. Optically identified X-ray sources from ROSAT Bright Source Catalogue (BSC; Voges et al. 1999) and Faint Source Catalogue (FSC; Voges et al. 2000) are included, 4253 X-ray selected AGN in total (56.2\% of all identified sources). All these sources are confirmed or candidate AGN based on Hamburg Quasar Survey (HQS) low-dispersion spectra. 3352 of them are listed in the Catalogue of QSOs and Active Galaxies (Véron-Cetty \& Véron (2010; 13th version) and 387 are in the Multifrequency Catalogue of Blazars (Roma-BZCAT) by Massaro et al. (2015).

We carried out classification for 210 of these candidate sources based on available SDSS spectra and enlarged the sample of confirmed AGN to 3650. A special emphasis is made on narrow-line Sy1.0-Sy1.5 galaxies and QSOs, as many of them have soft X-ray, strong FeII lines, and relatively narrow lines coming from the Broad Line Region (BLR; "narrow broad lines"); we have classified 45 new AGN as such objects.

We carried out statistical investigations of the sample, including study of the luminosity function, flux-ratios for different ranges, luminosity evolution, etc.

Multiwavelength SEDs have also been constructed based on photometric points from gamma-ray to radio to follow their behavior for different kinds of AGN and link these SEDs to classifications. AGN and their candidates were grouped into subsamples showing similar features (spectral index, etc.) and these subsamples were compared to known spectral classes. The sample is a relevant sources for identification of new blazars.

\section{References}

Massaro, E., Maselli, A., Leto, C., et al. 2015, ApछSSS, 357, 75

Mickaelian, A. M., Hovhannisyan, L. R., Engels, D., et al. 2006, A\&3 A, 449, 425

Véron-Cetty, M. P. \& Véron, P. 2010, A\& A, 518, A10

Voges, W., Aschenbach, B., Boller, Th., et al. 1999, A\& A, 349, 389

Voges, W., Aschenbach, B., Boller, Th., et al. 2000, IAU Circ., 7432R

Zickgraf, F.-J., Engels, D., Hagen, H.-J., Reimers, D., \& Voges, W. 2003, AËA, 406, 535 